\title{
On the Hausdorff dimension of a family of self-similar sets with complicated overlaps
}

by

\author{
Balázs Bárány (Budapest)
}

\begin{abstract}
We investigate the properties of the Hausdorff dimension of the attractor of the iterated function system (IFS) $\{\gamma x, \lambda x, \lambda x+1\}$. Since two maps have the same fixed point, there are very complicated overlaps, and it is not possible to directly apply known techniques. We give a formula for the Hausdorff dimension of the attractor for Lebesgue almost all parameters $(\gamma, \lambda), \gamma<\lambda$. This result only holds for almost all parameters: we find a dense set of parameters $(\gamma, \lambda)$ for which the Hausdorff dimension of the attractor is strictly smaller.
\end{abstract}

1. Introduction and statements of results. Our paper is motivated by a question of Pablo Shmerkin at the conference in Greifswald in 2008. The question was the following:

Question. What is the Hausdorff dimension of the attractor generated by the IFS $\left\{\frac{1}{4} x, \frac{1}{3} x, \frac{1}{3} x+\frac{2}{3}\right\}$ ?

Let us denote the Hausdorff dimension of a compact subset $\Lambda$ of $\mathbb{R}$ by $\operatorname{dim}_{\mathrm{H}} \Lambda$. For the definition and basic properties of Hausdorff dimension we refer the reader to [1] or [2]. Let us recall here the definition of the attractor.

Let $\left\{f_{0}, \ldots, f_{n}\right\}$ be a family of continuous self-maps on the real line. We will in addition assume that each $f_{i}$ is a contraction, that is, $\left|f_{i}(x)-f_{i}(y)\right| \leq$ $r_{i}|x-y|$ for all $x, y$ and some $0<r_{i}<1$. Then there exists a unique, nonempty compact subset $\Lambda$ of $\mathbb{R}$ which satisfies

$$
\Lambda=\bigcup_{k=0}^{n} f_{k}(\Lambda) .
$$

We call it the attractor of the iterated function system (IFS) $\left\{f_{0}(x), f_{1}(x), \ldots\right.$ $\left.\ldots, f_{n}(x)\right\}$. 
Let us suppose that the functions of the IFS are similarities of the form $\left\{f_{i}(x)=\lambda_{i} x+d_{i}\right\}_{i=0}^{n}$, where $0<\left|\lambda_{i}\right|<1$ for every $i \in\{0, \ldots, n\}$. We say that the attractor or the IFS itself is self-similar. It is well known that if a self-similar IFS satisfies the so-called open set condition (OSC), i.e. there exists an open set $U$ such that for every $i, j \in\{0, \ldots, n\}, f_{i}(U) \subset U$ and $f_{i}(U) \cap f_{j}(U)=\emptyset$ if $i \neq j$, then the Hausdorff dimension of the attractor is the unique solution $s$ of

$$
\sum_{i=0}^{n}\left|\lambda_{i}\right|^{s}=1
$$

(see for example [4]). Even if the OSC does not hold, the solution of (1.1) is called the similarity dimension of the IFS. The similarity dimension is always an upper bound for the Hausdorff dimension of the attractor (see [1]). In the case when the IFS has overlapping structure, i.e. the open set condition does not hold, the Hausdorff dimension of the attractor $\Lambda$ of the IFS $\left\{f_{i}(x)=\lambda_{i} x+d_{i}\right\}_{i=0}^{n}$ is

$$
\operatorname{dim}_{\mathrm{H}} \Lambda=\min \{1, s\} \quad \text { for a.e. } \mathbf{d} \in \mathbb{R}^{n+1},
$$

where $s$ is the unique solution of (1.1) (see [11] and [3]).

In this article, we consider the IFS $\{\gamma x, \lambda x, \lambda x+1\}$, where we assume that $0<\gamma<\lambda<1$. Let us denote the attractor of this IFS by $\Lambda^{\gamma, \lambda}$. The problem of calculating its Hausdorff dimension is far from being simple.

A special property of our class of IFS is that the first two maps have a common fixed point. This implies that they commute, so we observe an immense (increasing exponentially under iteration) amount of exact and partial overlaps in our system. Needless to say, the OSC does not hold.

Iterated function systems that do not satisfy OSC were first studied in [9], where the transversality condition method was first introduced. See [7], [8] for the most general treatment of this approach. Since that time, several other methods have been proposed: weak separation condition, finite type condition and others (see, for example, [5], [6] and [14]). However, neither of those is going to work for overlaps as severe as our system has.

For this reason, we are forced to modify the transversality method, applying it only to some subsystems of the IFS (details will be presented in the following sections). The main result of this paper is as follows:

TheOREM 1.1. Let $\Lambda^{\gamma, \lambda}$ be the attractor of the IFS $\{\gamma x, \lambda x, \lambda x+1\}$. Then for Lebesgue almost every $0<\gamma<\lambda<1 / 2$,

$$
\operatorname{dim}_{\mathrm{H}} \Lambda^{\gamma, \lambda}=\min \left\{1, s^{\gamma, \lambda}\right\},
$$

where $s^{\gamma, \lambda}$ is the unique solution of

$$
2 \lambda^{s}+\gamma^{s}-\lambda^{s} \gamma^{s}=1 .
$$


Moreover, $\mathcal{L}\left(\Lambda^{\gamma, \lambda}\right)>0$ for Lebesgue almost every $(\gamma, \lambda)$ such that $s^{\gamma, \lambda}>1$. More precisely, the above statements are true for every fixed $0<\lambda<1 / 2$ and Lebesgue almost every $0<\gamma<\lambda$.

Note that the assumption $\lambda<1 / 2$ is not really restrictive: the attractor of our system contains the attractor of its subsystem $\{\lambda x, \lambda x+1\}$, which for $\lambda \geq 1 / 2$ is an interval and, in particular, it has dimension 1 .

The equality (13) only holds for almost all parameters. And indeed, we can exhibit a family of parameter values for which it does not hold.

Proposition 1.2. Let $q$ and $p$ be integers and $q>p,(q, p)=1$. Let $\Lambda^{\lambda, q, p}$ be the attractor of $\left\{\lambda^{q / p} x, \lambda x, \lambda x+1\right\}$. Then

$$
\operatorname{dim}_{\mathrm{H}} \Lambda^{\lambda, q, p} \leq \min \left\{1, s_{p, q}^{\lambda}\right\},
$$

where $s_{p, q}^{\lambda}$ is the unique solution of

$$
2 \lambda^{s}+\sum_{k=1}^{p-1} \lambda^{(q k / p+1) s}=1 .
$$

Note that this family of exceptional parameter values is dense in $\{(\gamma, \lambda)$ : $2 \lambda+\gamma<1, \gamma<\lambda\}$, where the statement of Proposition 1.2 excludes the possibility that the equality of Theorem 1.1 holds. This implies that the function $(\gamma, \lambda) \mapsto \operatorname{dim}_{\mathrm{H}} \Lambda^{\gamma, \lambda}$ cannot be continuous.

2. Transversality methods. First let us introduce the transversality condition for self-similar IFS with one parameter. The definition corresponds to the definition in [12], [13], which was introduced for general IFS.

Let $U$ be an open, bounded interval of $\mathbb{R}$ and $\Sigma$ a finite set of symbols. Let $\Psi_{t}=\left\{\psi_{i}^{t}(x)=\lambda_{i}(t) x+d_{i}(t)\right\}_{i \in \Sigma}$, where $\lambda_{i}, d_{i} \in C^{1}(\bar{U})$ and $0<\alpha \leq$ $\lambda_{i}(t) \leq \beta<1$ for every $i \in \Sigma$ and $t \in \bar{U}$ and for some $\alpha, \beta \in(0,1)$. Let $\Lambda^{t}$ be the attractor of $\Psi_{t}$ and $\pi_{t}$ be the natural projection from the symbolic space $\Sigma^{\mathbb{N}}$ to $\Lambda^{t}$. More precisely, let $\mathbf{i}=\left(i_{0} i_{1} \ldots\right) \in \Sigma^{\mathbb{N}}$ and

$$
\pi_{t}(\mathbf{i})=\lim _{n \rightarrow \infty} \psi_{i_{0}}^{t} \circ \psi_{i_{1}}^{t} \circ \cdots \circ \psi_{i_{n}}^{t}(0) .
$$

It is well known that the limit exists and is independent of the base point 0 . Moreover, $\pi_{t}$ is a surjective function from $\Sigma^{\mathbb{N}}$ onto $\Lambda^{t}$. Denote by $\sigma$ the left-shift operator on $\Sigma^{\mathbb{N}}$. More precisely, let $\sigma:\left(i_{0} i_{1} \ldots\right) \mapsto\left(i_{1} i_{2} \ldots\right)$. It is easy to see that

$$
\pi_{t}(\mathbf{i})=\psi_{i_{0}}^{t}\left(\pi_{t}(\sigma \mathbf{i})\right) .
$$

DeFinition 2.1. We say that $\Psi_{t}$ satisfies the transversality condition on an open, bounded interval $U \subset \mathbb{R}$ if there exists a constant $C>0$ such that for every $\mathbf{i}, \mathbf{j} \in \Sigma^{\mathbb{N}}$ with $i_{0} \neq j_{0}$,

$$
\mathcal{L}\left(\left\{t \in U:\left|\pi_{t}(\mathbf{i})-\pi_{t}(\mathbf{j})\right| \leq r\right\}\right) \leq C r \quad \text { for every } r>0,
$$

where $\mathcal{L}$ is the Lebesgue measure on the real line. 
This definition is equivalent to the ones given in e.g. [12], [13]. Now let us recall the theorem of K. Simon, B. Solomyak and M. Urbański [12, Theorem 3.1] in the self-similar case with one parameter.

Theorem 2.2 (Simon, Solomyak, Urbański). Suppose that $\Psi_{t}$ satisfies the transversality condition on an open, bounded interval $U$. Then

(1) $\operatorname{dim}_{\mathrm{H}} \Lambda^{t}=\min \{s(t), 1\}$ for Lebesgue a.e. $t \in U$,

(2) $\mathcal{L}\left(\Lambda^{t}\right)>0$ for Lebesgue a.e. $t \in U$ such that $s(t)>1$,

where $s(t)$ is the similarity dimension of $\Psi_{t}$. More precisely, $s(t)$ satisfies the equation

$$
\sum_{i \in \Sigma} \lambda_{i}(t)^{s(t)}=1
$$

To prove transversality we can use the following lemma which follows from [12, Lemma 7.3].

Lemma 2.3. Let $U \subset \mathbb{R}$ be an open, bounded interval and $f_{\mathbf{i}, \mathbf{j}}(t)=\pi_{t}(\mathbf{i})$ $\pi_{t}(\mathbf{j})$. If for every $\mathbf{i}, \mathbf{j} \in \Sigma^{\mathbb{N}}$ with $i_{0} \neq j_{0}$ and for every $t_{0} \in U$,

$$
f_{\mathbf{i}, \mathbf{j}}\left(t_{0}\right)=0 \Rightarrow\left|\frac{d f_{\mathbf{i}, \mathbf{j}}}{d t}\left(t_{0}\right)\right|>0
$$

then there is transversality on any open interval $V$ whose closure is contained in $U$.

3. Proofs. Let us fix a $\lambda \in(0,1 / 2)$. Without loss of generality we can assume that $\gamma=c \lambda$, where $0<c<1$. Let $\psi_{0}^{c}(x)=c \lambda x, \psi_{1}^{c}(x)=\lambda x$ and $\psi_{2}^{c}(x)=\lambda x+1$. We note that $\psi_{1}^{c}, \psi_{2}^{c}$ do not depend on $c$. Let us define $\Sigma^{*}=\bigcup_{n=1}^{\infty} \Sigma^{n}$ and for every $n \geq 1$ let $\psi_{\underline{i}}^{c}=\psi_{i_{0}}^{c} \circ \psi_{i_{1}}^{c} \circ \cdots \circ \psi_{i_{n}}^{c}$ where $\underline{i} \in \Sigma^{n}$.

We note that $\Psi^{c}=\left\{\psi_{0}^{c}, \psi_{1}^{c}, \psi_{2}^{c}\right\}$ does not satisfy the transversality condition, since for every $\mathbf{i}, \mathbf{j} \in\{0,1\}^{\mathbb{N}}$ and $0<c<1$ we have $\pi_{c}(\mathbf{i})-\pi_{c}(\mathbf{j}) \equiv 0$. In order to prove Theorem 1.1, we are going to introduce well-chosen systems $\Psi_{n}^{c}$ which do satisfy transversality. Moreover, we are going to show that the attractor of $\Psi_{n}^{c}$ is contained in, and has dimension arbitrarily close to, the attractor $\Lambda^{c \lambda, \lambda}$ of the IFS $\Psi^{c}$, so that we are able to deduce information on the dimension of $\Lambda^{c \lambda, \lambda}$ by studying these subsystems with transversality.

First of all, we have to consider some properties of the natural projection. Let $\mathbf{i} \in \Sigma^{\mathbb{N}}$ and denote by $\mathbf{i}(n)$ the first $n$ elements of $\mathbf{i}$, and by $\sharp_{i} \mathbf{i}(n)$ the number of $i$ 's in $\mathbf{i}(n)$. Similarly, let $\sharp_{i} \mathbf{i}(n, l)$ be the number of $i$ 's between the $n$th and $l$ th elements of $\mathbf{i}$. We note that $\mathbf{i}(0)$ is the empty word and $\sharp_{i} \mathbf{i}(-1, l):=\sharp_{i} \mathbf{i}(l)$. Then

$$
\pi_{c}(\mathbf{i})=\sum_{k=0}^{\infty} \delta_{i_{k}} c^{\sharp_{0}} \mathbf{i}(k) \lambda^{k},
$$


where $\delta_{i}=1$ if $i=2$, else $\delta_{i}=0$. We will write this natural projection in another form.

Let $\alpha_{2}^{\mathbf{i}}$ be the number of 2's in i, more precisely, let $\alpha_{2}^{\mathbf{i}}=\lim _{n \rightarrow \infty} \sharp_{2} \mathbf{i}(n)$ if $\mathbf{i}$ is an infinite length word, and $\alpha_{2}^{\mathbf{i}}=\sharp_{2} \mathbf{i}(|\mathbf{i}|)$ if $\mathbf{i}$ is finite. If $\mathbf{i}$ is infinite then $\alpha_{2}^{\mathbf{i}}$ can be infinity. We will write $n_{l}^{\mathbf{i}}$ for the place of the $l$ th 2 in $\mathbf{i}$ (here $\mathbf{i}$ can be finite or infinite) for every $1 \leq l \leq \alpha_{2}^{\mathbf{i}}$. We define $n_{0}^{\mathbf{i}}:=-1$, and if $\alpha_{2}^{\mathbf{i}}$ is finite, then let $n_{\alpha_{2}^{\mathbf{i}}+1}^{\mathbf{i}}:=|\mathbf{i}|$. We note that if $\mathbf{i}$ does not contain any 2 's then $\pi_{c}(\mathbf{i})=0$.

Observe that with this notation, $\pi_{c}$ can be written as

$$
\pi_{c}(\mathbf{i})=\sum_{k=1}^{\alpha_{2}^{\mathbf{i}}} c^{\sharp_{0}} \mathbf{i}\left(n_{k}^{\mathbf{i}}\right) \lambda^{n_{k}^{\mathbf{i}},}
$$

where an empty sum is defined to be 0 .

If $\alpha_{2}^{\mathbf{i}} \neq 0$, then let $P_{\mathbf{i}}=\left\{\#_{0} \mathbf{i}\left(n_{l}^{\mathbf{i}}\right): l \geq 1\right\}$, else let $P_{\mathbf{i}}=\emptyset$. Moreover, let

$$
m_{k}^{\mathbf{i}}=\sup \left\{j: \sharp_{0} \mathbf{i}\left(n_{j}^{\mathbf{i}}\right)=k\right\}, \quad r_{k}^{\mathbf{i}}=\inf \left\{j: \sharp_{0} \mathbf{i}\left(n_{j}^{\mathbf{i}}\right)=k\right\}
$$

for $k \in P_{\mathbf{i}}$. We note that $P_{\mathbf{i}}$ can be finite or infinite, and if it is finite then $m_{\max P_{\mathrm{i}}}^{\mathrm{i}}$ can be infinity. Therefore

$$
\pi_{c}(\mathbf{i})=\sum_{k \in P_{\mathbf{i}}} c^{k} d_{k}^{\mathbf{i}}(\lambda), \quad \text { where } \quad d_{k}^{\mathbf{i}}(\lambda)=\sum_{l=r_{k}^{\mathbf{i}}}^{m_{k}^{\mathbf{i}}} \lambda^{n_{l}^{\mathbf{i}}} .
$$

We call $n_{m_{k}^{\mathbf{i}}}^{\mathbf{i}}$ the degree and $n_{r_{k}^{\mathbf{i}}}^{\mathbf{i}}$ the lower degree of $d_{k}^{\mathbf{i}}(\lambda)$, denoted by deg and lowerdeg respectively.

Lemma 3.1. For every $\mathbf{i} \in \Sigma^{\mathbb{N}}$ and $\lambda \in(0,1 / 2)$ we have

(1) lowerdeg $d_{k}^{\mathbf{i}}(\lambda) \geq k$ for every $k \in P_{\mathbf{i}}$,

(2) $\operatorname{deg} d_{k}^{\mathbf{i}}(\lambda)+l-k+1 \leq \operatorname{lowerdeg} d_{l}^{\mathbf{i}}(\lambda)$ for every $k, l \in P_{\mathbf{i}}, k<l$,

(3) $\lambda^{l-k} d_{k}^{\mathbf{i}}(\lambda) \geq d_{l}^{\mathbf{i}}(\lambda)$ for every $k, l \in P_{\mathbf{i}}, k<l$.

Proof. Since $\sharp_{0} \mathbf{i}(k) \leq k,(1)$ is obvious.

Let $k, l \in P_{\mathbf{i}}$ with $k<l$. Since $\operatorname{deg} d_{k}^{\mathbf{i}}(\lambda)=n_{m_{k}^{\mathbf{i}}}^{\mathbf{i}}$ and lowerdeg $d_{l}^{\mathbf{i}}(\lambda)=n_{r_{l}^{\mathbf{i}}}^{\mathbf{i}}$, between the $n_{m_{k}^{\mathrm{i}}}^{\mathbf{i}}$ th and $n_{r_{l}^{\mathrm{i}}}^{\mathbf{i}}$ th elements of $\mathbf{i}$ there have to be $l-k$ zeros. Therefore

$$
l-k=\sharp_{0} \mathbf{i}\left(n_{m_{k}^{\mathbf{i}}}^{\mathbf{i}}, n_{r_{l}^{\mathbf{i}}}^{\mathbf{i}}\right) \leq n_{r_{l}^{\mathbf{i}}}^{\mathbf{i}}-n_{m_{k}^{\mathbf{i}}}^{\mathbf{i}}-1 .
$$

This completes the proof of (2). The property (3) is an easy consequence of (2) by using the fact that $\lambda<1 / 2$.

Now we are going to define the families $\Psi_{n}^{c}$ of iterated function systems for which transversality holds. First of all, we define sets $\Sigma_{i}$ of finite length 
words by induction. Let $\Sigma_{1}=\{1,2\}$ and for every $n \geq 1$ let

$$
\Sigma_{n+1}=\bigcup_{\mathbf{i} \in \Sigma_{n}, i_{0} \neq 1}\{0 \mathbf{i}\} \cup \bigcup_{\mathbf{i} \in \Sigma_{n}}\{1 \mathbf{i}, 2 \mathbf{i}\} .
$$

For example $\Sigma_{2}=\{1,2\}^{2} \cup\{02\}$ and $\Sigma_{3}=\{1,2\}^{3} \cup\{102,202,021,022,002\}$ etc. Obviously, $\Sigma_{n} \subset \Sigma^{n}$ for every $n \geq 1$.

Lemma 3.2. For every $n \geq 1$ and every $\underline{i}, \underline{j} \in \Sigma_{n}, \underline{i}=\underline{j}$ if and only if

$$
\begin{aligned}
& \alpha_{2}^{\underline{i}}=\alpha_{2}^{\frac{j}{2}}, \\
& \forall 0 \leq l \leq \alpha_{2}^{\frac{i}{2}}=\alpha_{2}^{\frac{j}{2}}, \quad n_{l} \underline{\underline{i}}=n_{\bar{l}}^{j} \quad \text { and } \quad \sharp_{0} \underline{i}\left(n_{l}^{\underline{i}}, n_{l+1}^{\underline{i}}\right)=\sharp_{0} \underline{j}\left(n_{\bar{l}}, n_{\bar{l}+1}^{j}\right) .
\end{aligned}
$$

Proof. The implication $(\underline{i}=\underline{j}) \Rightarrow(3.5)$ is obvious for every $n \geq 1$. We prove the other direction by induction.

For $n=1,(3.5) \Rightarrow(\underline{i}=\underline{j})$ is trivial. Suppose that $\Sigma_{n}$ satisfies the statement and let $\underline{i}, j \in \Sigma_{n+1}$ be such that $\underline{i}, j$ satisfy (3.5). Then either $\underline{i}=2 \underline{i}^{\prime}$ and $\underline{j}=2 \underline{j}^{\prime}$, or $\underline{i}=0 \underline{i}^{\prime}$ or $1 \underline{i}^{\prime}$ and $\underline{j}=0 \underline{j}^{\prime}$ or $1 \underline{j}^{\prime}$, where $\underline{i}^{\prime}, \underline{j}^{\prime} \in \Sigma_{n}$. In the first case we have $\underline{i}=j$ by the induction assumption.

In the second case we will show that $\underline{i}=0 \underline{i}^{\prime}$ if and only if $\underline{j}=0 \underline{j}$. Let us suppose that $\underline{i}=0 \underline{i}^{\prime}$. Then

$$
\sharp_{0} \underline{j}\left(n_{1}^{\underline{j}}\right)=\sharp_{0} \underline{i}\left(n_{1}^{\underline{i}}\right)=n_{1}^{\underline{i}}=n_{1}^{\underline{j}} .
$$

In the middle equality we have used (3.4). Therefore $j=0 j^{\prime}$. The reverse direction is similar. By using the induction assumption we have $\underline{i}=\underline{j}$.

Lemma 3.3. For every arbitrarily small $\varepsilon>0$ and every $n \geq 2$ the system $\Psi_{n}^{c}=\left\{\psi_{\underline{i}}^{c}\right\}_{\underline{i} \in \Sigma_{n}}$ satisfies the transversality condition for $c \in(\varepsilon, 1-\varepsilon)$.

Proof. We note that $\lambda \in(0,1 / 2)$ is fixed. Let $\varepsilon>0$ be arbitrarily small but fixed. We are going to prove transversality of $\Psi_{n}^{c}$ by using Lemma 2.3. More precisely, we are going to show that $(2.2)$ holds on $U=(\varepsilon / 2,1)$.

Suppose that $c \in(\varepsilon / 2,1)$. Let $\widetilde{\mathbf{i}}, \tilde{\mathbf{j}} \in \Sigma_{n}^{\mathbb{N}}$ be such that $\widetilde{\mathbf{i}}=\left(\underline{i}_{0} \underline{i}_{1} \underline{i}_{2} \ldots\right)$, $\widetilde{\mathbf{j}}=\left(\underline{j}_{0} \underline{j}_{1} \underline{j}_{2} \ldots\right)$ and $\underline{i}_{0} \neq \underline{j}_{0}$. We can define the natural projection of $\Psi_{n}^{c}=$ $\left\{\psi_{\underline{i}}^{c}\right\}_{\underline{i} \in \Sigma_{n}}$ as in (2.1). Denote it by $\widetilde{\pi}_{c}^{n}$.

Assume that $\left.\widetilde{\pi}_{c_{0}}^{n} \widetilde{\mathbf{i}}\right)=\widetilde{\pi}_{c_{0}}^{n}(\widetilde{\mathbf{j}})$ for a $c_{0} \in(\varepsilon / 2,1)$. Let $\mathbf{i}=\widetilde{\mathbf{i}}, \mathbf{j}=\widetilde{\mathbf{j}}$ as elements of $\Sigma^{\mathbb{N}}$. Then $\widetilde{\pi}_{c}^{n}(\widetilde{\mathbf{i}})=\pi_{c}(\mathbf{i})$ and $\widetilde{\pi}_{c}^{n}(\widetilde{\mathbf{j}})=\pi_{c}(\mathbf{j})$.

If $\min P_{\mathbf{i}}=\min P_{\mathbf{j}}$ the numbers of zeros before the $n_{1}^{\mathbf{i}}$ th element of $\mathbf{i}$ and before the $n_{1}^{\mathbf{j}}$ th element of $\mathbf{j}$ are the same. If $n_{1}^{\mathbf{i}}>n_{1}^{\mathbf{j}}$ then some simple algebra, using $\lambda<1 / 2$, shows that $\pi_{c_{0}}(\mathbf{i})<\pi_{c_{0}}(\mathbf{j})$, which is a contradiction. Likewise, we cannot have $n_{1}^{\mathbf{i}}<n_{1}^{\mathbf{j}}$, so necessarily $n_{1}^{\mathbf{i}}=n_{1}^{\mathbf{j}}$.

However, if $n_{1}^{\mathbf{i}}=n_{1}^{s}$, then

$$
\pi_{c_{0}}(\mathbf{i})-\pi_{c_{0}}(\mathbf{j})=c^{\min P_{\mathbf{i}}} \lambda^{n_{1}^{\mathbf{i}}}\left(\pi_{c_{0}}\left(\sigma^{n_{1}^{\mathbf{j}}+1} \mathbf{i}\right)-\pi_{c_{0}}\left(\sigma^{n_{1}^{\mathbf{j}}+1} \mathbf{j}\right)\right),
$$

where $\sigma$ is the left-shift operator on $\Sigma^{\mathbb{N}}=\{0,1,2\}^{\mathbb{N}}$. 
Since we have supposed that $\underline{i}_{0} \neq \underline{j}_{0} \in \Sigma_{n}$, by Lemma 3.2 and $c>\varepsilon / 2$ we can assume without loss of generality that $\min P_{\mathbf{i}}>\min P_{\mathbf{j}}$. Let $l_{\mathbf{j}}=\min P_{\mathbf{j}}$. Then

$$
\begin{aligned}
f_{\mathbf{j}, \mathbf{i}}(c) & \left.=\widetilde{\pi}_{c}^{n} \widetilde{\mathbf{j}}\right)-\widetilde{\pi}_{c}^{n}(\widetilde{\mathbf{i}})=\pi_{c}(\mathbf{j})-\pi_{c}(\mathbf{i}) \\
& =c^{l_{\mathbf{j}}} d_{l_{\mathbf{j}}}^{\mathbf{j}}(\lambda)+\sum_{k \in P_{\mathbf{j}} \backslash\left\{l_{\mathbf{j}}\right\}} c^{k} d_{k}^{\mathbf{j}}(\lambda)-\sum_{k \in P_{\mathbf{i}}} c^{k} d_{k}^{\mathbf{i}}(\lambda) \\
& =c^{l_{\mathbf{j}}} d_{l_{\mathbf{j}}}^{\mathbf{j}}(\lambda)\left(1+\sum_{k \in P_{\mathbf{j}} \backslash\left\{l_{\mathbf{j}}\right\}} c^{k-l_{\mathbf{j}}} \frac{d_{k}^{\mathbf{j}}(\lambda)}{d_{l_{\mathbf{j}}}^{\mathbf{j}}(\lambda)}-\sum_{k \in P_{\mathbf{i}}} c^{k-l_{\mathbf{j}}} \frac{d_{k}^{\mathbf{i}}(\lambda)}{d_{l_{\mathbf{j}}}^{\mathbf{j}}(\lambda)}\right) .
\end{aligned}
$$

Define

$$
g_{\mathbf{j}, \mathbf{i}}(c)=1+\sum_{k \in P_{\mathbf{j}} \backslash\left\{l_{\mathbf{j}}\right\}} c^{k-l_{\mathbf{j}}} \frac{d_{k}^{\mathbf{j}}(\lambda)}{d_{l_{\mathbf{j}}}^{\mathbf{j}}(\lambda)}-\sum_{k \in P_{\mathbf{i}}} c^{k-l_{\mathbf{j}}} \frac{d_{k}^{\mathbf{i}}(\lambda)}{d_{l_{\mathbf{j}}}^{\mathbf{j}}(\lambda)} .
$$

Note that since $c>\varepsilon / 2>0$ one has $f_{\mathbf{j}, \mathbf{i}}\left(c_{0}\right)=0$ if and only if $g_{\mathbf{j}, \mathbf{i}}\left(c_{0}\right)=0$. On the other hand, it is easy to see that if $g_{\mathbf{j}, \mathbf{i}}\left(c_{0}\right)=0$, then

$$
\frac{d f_{\mathbf{j}, \mathbf{i}}}{d c}\left(c_{0}\right)=0 \Leftrightarrow \frac{d g_{\mathbf{j}, \mathbf{i}}}{d c}\left(c_{0}\right)=0 .
$$

Therefore it is enough to prove

$$
\frac{d g_{\mathbf{j}, \mathbf{i}}}{d c}(c)=0 \Rightarrow g_{\mathbf{j}, \mathbf{i}}(c)>0 .
$$

So suppose that $\frac{d g_{\mathbf{j}, \mathbf{i}}}{d c}\left(c_{0}\right)=0$ for some $c_{0} \in(\varepsilon / 2,1)$. Then

$$
\begin{aligned}
0 & =c_{0} \frac{d g_{\mathbf{j}, \mathbf{i}}}{d c}\left(c_{0}\right) \\
& =c_{0}\left(\sum_{k \in P_{\mathbf{j}} \backslash\left\{l_{\mathbf{j}}\right\}}\left(k-l_{\mathbf{j}}\right) c_{0}^{k-l_{\mathbf{j}}-1} \frac{d_{k}^{\mathbf{j}}(\lambda)}{d_{l_{\mathbf{j}}}^{\mathbf{j}}(\lambda)}-\sum_{k \in P_{\mathbf{i}}}\left(k-l_{\mathbf{j}}\right) c_{0}^{k-l_{\mathbf{j}}-1} \frac{d_{k}^{\mathbf{i}}(\lambda)}{d_{l_{\mathbf{j}}}^{\mathbf{j}}(\lambda)}\right) \\
& \leq \sum_{k \in P_{\mathbf{j}} \backslash\left\{l_{\mathbf{j}}\right\}}\left(k-l_{\mathbf{j}}\right) c_{0}^{k-l_{\mathbf{j}}} \frac{d_{k}^{\mathbf{j}}(\lambda)}{d_{l_{\mathbf{j}}}^{\mathbf{j}}(\lambda)}-\sum_{k \in P_{\mathbf{i}}} c_{0}^{k-l_{\mathbf{j}}} \frac{d_{k}^{\mathbf{i}}(\lambda)}{d_{l_{\mathbf{j}}}^{\mathbf{j}}(\lambda)} \\
& =\sum_{k \in P_{\mathbf{j}} \backslash\left\{l_{\mathbf{j}}\right\}}\left(k-l_{\mathbf{j}}-1\right) c_{0}^{k-l_{\mathbf{j}}} \frac{d_{k}^{\mathbf{j}}(\lambda)}{d_{l_{\mathbf{j}}}^{\mathbf{j}}(\lambda)}+\sum_{k \in P_{\mathbf{j}} \backslash\left\{l_{\mathbf{j}}\right\}} c_{0}^{k-l_{\mathbf{j}}} \frac{d_{k}^{\mathbf{j}}(\lambda)}{d_{l_{\mathbf{j}}}^{\mathbf{j}}(\lambda)}-\sum_{k \in P_{\mathbf{i}}} c_{0}^{k-l_{\mathbf{j}}} \frac{d_{k}^{\mathbf{i}}(\lambda)}{d_{l_{\mathbf{j}}}^{\mathbf{j}}(\lambda)} .
\end{aligned}
$$

By using (3) of Lemma 3.1 we have

$$
0 \leq \sum_{k \in P_{\mathbf{j}} \backslash\left\{l_{\mathbf{j}}\right\}}\left(k-l_{\mathbf{j}}-1\right) c_{0}^{k-l_{\mathbf{j}}} \lambda^{k-l_{\mathbf{j}}}+\sum_{k \in P_{\mathbf{j}} \backslash\left\{l_{\mathbf{j}}\right\}} c_{0}^{k-l_{\mathbf{j}}} \frac{d_{k}^{\mathbf{j}}(\lambda)}{d_{l_{\mathbf{j}}}^{\mathbf{j}}(\lambda)}-\sum_{k \in P_{\mathbf{i}}} c_{0}^{k-l_{\mathbf{j}}} \frac{d_{k}^{\mathbf{i}}(\lambda)}{d_{l_{\mathbf{j}}}^{\mathbf{j}}(\lambda)}
$$


We can give an upper bound for the first term:

$$
\sum_{k \in P_{\mathbf{j}} \backslash\left\{l_{\mathbf{j}}\right\}}\left(k-l_{\mathbf{j}}-1\right) c_{0}^{k-l_{\mathbf{j}}} \lambda^{k-l_{\mathbf{j}}} \leq \sum_{k=1}^{\infty}(k-1) c_{0}^{k} \lambda^{k}=\frac{\left(c_{0} \lambda\right)^{2}}{\left(1-c_{0} \lambda\right)^{2}}<1 .
$$

In the last inequality we have used that $\lambda<1 / 2$. Therefore $0<g_{\mathbf{j}, \mathbf{i}}\left(c_{0}\right)$ by the definition of $g_{\mathbf{j}, \mathbf{i}}(c)$.

Using Lemma 2.3 for $U=(\varepsilon / 2,1)$ we conclude that $\Psi_{n}^{c}$ satisfies transversality on $V=(\varepsilon, 1-\varepsilon)$.

Let us denote the attractor of $\Psi_{n}^{c}=\left\{\psi_{\underline{i}}^{c}\right\}_{\underline{i} \in \Sigma_{n}}$ by $\Lambda_{\Sigma_{n}}^{c \lambda, \lambda}$.

Proposition 3.4. For every $0<\lambda<1 / 2$ and Lebesgue almost every $0<c<1$

$$
\lim _{n \rightarrow \infty} \operatorname{dim}_{\mathrm{H}} \Lambda_{\Sigma_{n}}^{c \lambda, \lambda}=\operatorname{dim}_{\mathrm{H}} \Lambda^{c \lambda, \lambda} .
$$

Proof. First we note that by Lemma 3.3 and Theorem 2.2, for every $\varepsilon>0, n \geq 1$ and almost every $\varepsilon<c<1-\varepsilon$ we have

$$
\operatorname{dim}_{\mathrm{H}} \Lambda_{\Sigma_{n}}^{c \lambda, \lambda}=\min \left\{1, s_{n}^{c, \lambda}\right\}
$$

where $s_{n}^{c, \lambda}$ is the unique solution of

$$
\sum_{\underline{i} \in \Sigma_{n}}\left(c^{\sharp} \underline{i}^{\underline{\lambda}} \lambda^{n}\right)^{s}=1 .
$$

Since for every $\varepsilon>0$ the dimension formula (3.6) holds for a.e. $c \in(\varepsilon, 1-\varepsilon)$, it holds for a.e. $c \in(0,1)$. We note that $s_{n}^{c, \lambda}$ is a bounded, increasing sequence, therefore it is convergent. Let $s_{c, \lambda}^{*}$ be its limit.

The lower bound is trivial since $\Lambda_{\Sigma_{n}}^{c \lambda, \lambda} \subseteq \Lambda^{c \lambda, \lambda}$ for every $n$. Therefore

$$
\min \left\{s_{c, \lambda}^{*}, 1\right\} \leq \operatorname{dim}_{\mathrm{H}} \Lambda^{c \lambda, \lambda}
$$

for every $0<\lambda<1 / 2$ and Lebesgue almost every $0<c<1$.

Now we prove the upper bound. It is easy to see that the convex hull of $\Lambda^{c \lambda, \lambda}$ is the interval $[0,1 /(1-\lambda)]$. By using the fact

$$
\psi_{0}^{c} \circ \psi_{1}^{c}(x) \equiv \psi_{1}^{c} \circ \psi_{0}^{c}(x)
$$

and $0<c<1$ we see that for every $\underline{i} \in \Sigma^{n}$ there exists $j \in \Sigma_{n}$ such that

$$
\psi_{\underline{i}}^{c}\left(\left[0, \frac{1}{1-\lambda}\right]\right) \subseteq \psi_{\underline{j}}^{c}\left(\left[0, \frac{1}{1-\lambda}\right]\right) .
$$

We indicate how to find such a $\underline{j} \in \Sigma_{n}$. The positions of the 2's in $\underline{j}$ are the same as in $\underline{i}$. Before the first appearance of 2 and between any two consecutive appearances of 2 , we keep the same number of 0 's as in $\underline{i}$, but change the order so that all 1's come before all 0's. After the last occurrence of a 2 (or everywhere if there are no 2's in $\underline{i}$ ), we replace all 0's by 1's. 
For each $n \in \mathbb{N}$, we consider the covering of the attractor $\Lambda^{c \lambda, \lambda}$ given by $\left\{\psi_{\underline{i}}^{c}([0,1 /(1-\lambda)])\right\}_{\underline{i} \in \Sigma_{n}}$, and note that the diameters of the sets in the cover are at most $\lambda^{n}$. Therefore by using the definition of Hausdorff measure (see [1]) we have

$$
\mathcal{H}_{\lambda^{n}}^{s}\left(\Lambda^{c \lambda, \lambda}\right) \leq \sum_{\underline{i} \in \Sigma_{n}}\left(\frac{c^{\sharp_{0}} \underline{\underline{i}} \lambda^{n}}{1-\lambda}\right)^{s},
$$

where $\mathcal{H}_{\delta}^{s}(\Lambda)=\inf \left\{\sum_{i}\left|U_{i}\right|^{s}: \Lambda \subset \bigcup_{i} U_{i},\left|U_{i}\right|<\delta\right\}$. Let $\varepsilon>0$ be arbitrarily small. Then

$$
\mathcal{H}_{\lambda^{n}}^{s_{c, \lambda}^{*}+\varepsilon}\left(\Lambda^{c \lambda, \lambda}\right) \leq \sum_{\underline{i} \in \Sigma_{n}}\left(\frac{c^{\sharp_{0}} \underline{\underline{i}} \lambda^{n}}{1-\lambda}\right)^{s_{c, \lambda}^{*}+\varepsilon} \leq \lambda^{n \varepsilon}\left(\frac{1}{1-\lambda}\right)^{s_{c, \lambda}^{*}+\varepsilon},
$$

which tends to 0 as $n \rightarrow \infty$. Therefore by the definition of Hausdorff dimension (see [1])

$$
\operatorname{dim}_{\mathrm{H}} \Lambda^{c \lambda, \lambda} \leq s_{c, \lambda}^{*}+\varepsilon
$$

where $\varepsilon>0$ is arbitrary. Thus the proposition is proved.

Now we are able to prove Theorem 1.1.

Proof of Theorem 1.1. Let $\widetilde{\Sigma}_{n}$ be the following set of symbols:

$$
\widetilde{\Sigma}_{n}=\{1,2,02,002, \ldots, \overbrace{0 \ldots 0}^{n-1} 2\} \text {. }
$$

Let $\Lambda_{\widetilde{\Sigma}_{n}}^{c \lambda, \lambda}$ be the attractor of $\left\{\psi_{\underline{i}}^{c}\right\}_{\underline{i} \in \widetilde{\Sigma}_{n}}$. Notice that every $\underline{i} \in \Sigma_{n}$ can be decomposed as a juxtaposition $\underline{i}=\underline{j}_{1} \ldots \underline{j}_{k}$, where each $\underline{j}_{r}$ is in $\widetilde{\Sigma}_{n}$. Therefore for every $0<\lambda<1 / 2$ and Lebesgue almost every $0<c<1, \Lambda_{\Sigma_{n}}^{c \lambda, \lambda} \subseteq \Lambda_{\widetilde{\Sigma}_{n}}^{c \lambda, \lambda}$, and hence by Proposition 3.4,

$$
\operatorname{dim}_{\mathrm{H}} \Lambda^{c \lambda, \lambda}=\lim _{n \rightarrow \infty} \operatorname{dim}_{\mathrm{H}} \Lambda_{\Sigma_{n}}^{c \lambda, \lambda} \leq \lim _{n \rightarrow \infty} \operatorname{dim}_{\mathrm{H}} \Lambda_{\widetilde{\Sigma}_{n}}^{c \lambda, \lambda} .
$$

The lower bound is trivial, therefore

$$
\operatorname{dim}_{\mathrm{H}} \Lambda^{c \lambda, \lambda}=\lim _{n \rightarrow \infty} \operatorname{dim}_{\mathrm{H}} \Lambda_{\widetilde{\Sigma}_{n}}^{c \lambda, \lambda} .
$$

We use the fact that for every $n \geq 2,\left\{\psi_{\underline{i}}^{c}\right\}_{\underline{i} \in \widetilde{\Sigma}_{n}}$ satisfies the transversality condition on $(\varepsilon, 1-\varepsilon)$ for all $\varepsilon>0$; as the proof of this claim is very similar to the proof of Lemma 3.3, we omit it. By Theorem 2.2 and by similar arguments to those at the beginning of the proof of Proposition 3.4, for every $n \geq 1$ and almost every $0<c<1$ we have

$$
\operatorname{dim}_{\mathrm{H}} \Lambda_{\widetilde{\Sigma}_{n}}^{c \lambda, \lambda}=\min \left\{1, \widetilde{s}_{n}^{c, \lambda}\right\}
$$

where $\widetilde{s}_{n}^{c, \lambda}$ is the unique solution of

$$
2 \lambda^{s}+\sum_{k=1}^{n-1}\left(c^{k} \lambda^{k+1}\right)^{s}=1
$$


It is easy to see by (3.8) that $s^{c \lambda, \lambda}=\lim _{n \rightarrow \infty} \widetilde{s}_{n}^{c, \lambda}$ is the unique solution of

$$
2 \lambda^{s}+\sum_{k=1}^{\infty}\left(c^{k} \lambda^{k+1}\right)^{s}=1
$$

We note that the function $f_{1}(s)=2 \lambda^{s}+\lambda^{s} \gamma^{s} /\left(1-\gamma^{s}\right)$ is strictly increasing for every $\gamma, \lambda \in(0,1)$, and moreover $\lim _{s \rightarrow 0+} f_{1}(s)=\infty$ and $\lim _{s \rightarrow \infty} f_{1}(s)=0$. Therefore the equation (3.9) has a unique solution $s$, which also satisfies

$$
2 \lambda^{s}+(c \lambda)^{s}-\left(c \lambda^{2}\right)^{s}=1 .
$$

By similar arguments one can prove that (3.10) has a unique solution as well, which is the first statement of Theorem 1.1.

Now we prove the measure claim of Theorem 1.1. If $s^{c \lambda, \lambda}>1$, then $\widetilde{s}_{n}^{c, \lambda}>1$ for large enough $n$, so that, by transversality, $\widetilde{\Lambda}_{\widetilde{\Sigma}_{n}}^{c \lambda, \lambda}$ has positive Lebesgue measure for almost every $c$. Since $\widetilde{\Lambda}_{\widetilde{\Sigma}_{n}}^{c \lambda, \lambda} \subset \Lambda^{c \lambda, \lambda}$, this completes the proof of Theorem 1.1.

Finally, we prove Proposition 1.2.

Proof of Proposition 1.2. Let $q, p$ be integers such that $(q, p)=1$ and $q>p$ and let $\psi_{0}(x)=\lambda^{q / p} x, \psi_{1}(x)=\lambda x, \psi_{2}(x)=\lambda x+1$. It is easy to see that

$$
\psi_{01}(x) \equiv \psi_{10}(x), \quad \psi_{p}^{0 \ldots 0}(x) \equiv \psi_{q}^{1 \ldots 1}(x) .
$$

Therefore for any $\mathbf{i} \in \Sigma^{\mathbb{N}}=\{0,1,2\}^{\mathbb{N}}$ we can choose $\mathbf{j} \in \widetilde{\Sigma}_{p}^{\mathbb{N}}$ (where $\widetilde{\Sigma}_{p}$ is defined as in (3.7)) such that

$$
\pi(\mathbf{i})=\pi(\mathbf{j}) .
$$

Indeed, whenever there are at least $p$ consecutive zeros in $\mathbf{i}$, we can replace each block of $p$ consecutive zeros by a block of $q$ consecutive ones, and then rearrange the zeros and ones between two consecutive twos, by moving the ones to the front. Therefore

$$
\operatorname{dim}_{\mathrm{H}} \Lambda^{\lambda, q, p}=\operatorname{dim}_{\mathrm{H}} \Lambda_{\widetilde{\Sigma}_{p}}^{\lambda, q, p}
$$

where $\Lambda_{\widetilde{\Sigma}_{p}}^{\lambda, q, p}$ is the attractor of the IFS $\left\{\psi_{\underline{i}}\right\}_{\underline{i} \in \widetilde{\Sigma}_{p}}$. Since the Hausdorff dimension of a self-similar set is always at most the minimum of the similarity dimension (see (1.1)) and the dimension of the ambient space, we have

$$
\operatorname{dim}_{\mathrm{H}} \Lambda_{\widetilde{\Sigma}_{p}}^{\lambda, q, p} \leq \min \left\{1, s_{q, p}^{\lambda}\right\}
$$

where $s_{q, p}^{\lambda}$ is the unique solution of

which was to be proved.

$$
2 \lambda^{s}+\sum_{k=1}^{p-1} \lambda^{(q k / p+1) s}=1,
$$


Acknowledgments. I am grateful to Pablo Shmerkin and Michał Rams for useful discussions.

This research was supported by the EU FP6 Research Training Network CODY.

\section{References}

[1] K. J. Falconer, Fractal Geometry: Mathematical Foundations and Applications, Wiley, 1990.

[2] - Techniques in Fractal Geometry, Wiley, 1997.

[3] - , The Hausdorff dimension of some fractals and attractors of overlapping construction, J. Statist. Phys. 42 (1987), 123-132.

[4] J. E. Hutchinson, Fractals and self-similarity, Indiana Univ. Math. J. 30 (1981), $713-747$.

[5] K.-S. Lau, S.-M. Ngai and H. Rao, Iterated function systems with overlaps and self-similar measures, J. London Math. Soc. (2) 63 (2001), 99-116.

[6] S.-M. Ngai and Y. Wang, Hausdorff dimension of self-similar sets with overlaps, J. London Math. Soc. (2) 63 (2001), 655-672.

[7] Y. Peres and B. Solomyak, Absolute continuity of Bernoulli convolutions, a simple proof, Math. Res. Lett. 3 (1996), 231-239.

[8] -, -, Self-similar measures and intersections of Cantor sets, Trans. Amer. Math. Soc. 350 (1998), 4065-4087.

[9] M. Pollicott and K. Simon, The Hausdorff dimension of $\lambda$-expansions with deleted digits, Trans. Amer. Math. Soc. 347 (1995), 967-983.

[10] H. Rao and Z.-Y. Wen, A class of self-similar fractals with overlap structure, Adv. Appl. Math. 20 (1998), 50-72.

[11] K. Simon and B. Solomyak, On the dimension of self-similar sets, Fractals 10 (2002), $59-65$.

[12] K. Simon, B. Solomyak and M. Urbański, Hausdorff dimension of limit sets for parabolic IFS with overlaps, Pacific J. Math. 201 (2001), 441-478.

[13] - , - - Invariant measures for parabolic IFS with overlaps and random continued fractions, Trans. Amer. Math. Soc. 353 (2001), 5145-5164.

[14] M. W. Zerner, Weak separation properties for self-similar sets, Proc. Amer. Math. Soc. 124 (1996), 3529-3539.

Balázs Bárány

Department of Stochastics

Institute of Mathematics

Technical University of Budapest

P.O. Box 91

1521 Budapest, Hungary

E-mail: balubsheep@gmail.com

Received 9 March 2009;

in revised form 9 July 2009 